PROCEEDINGS OF THE

AMERICAN MATHEMATICAL SOCIETY

Volume 139, Number 1, January 2011, Pages 129-137

S 0002-9939(2010)10411-2

Article electronically published on September 1,2010

\title{
REGULARITY OF RIGID CR HYPERSURFACES IN A SPHERE
}

\author{
SUNG-YEON KIM
}

(Communicated by Franc Forstneric)

\begin{abstract}
Let $M$ be a germ of a smooth CR manifold of hypersurface type of dimension $2 n+1$ in a sphere $S$ of dimension $2 N+1$ with $n<N$. In this paper, we show that if $M$ is rigid and if $N-n<n / 2$, then there exists a complex manifold $V$ of (complex) dimension $n+1$ intersecting $S$ transversally such that $M=S \cap V$. As a consequence, we show that $M$ is real analytic.
\end{abstract}

\section{INTRODUCTION}

Let $M$ be a smooth real manifold of dimension $2 n+1$ in $\mathbb{C}^{N+1}$ with $1 \leq n \leq N$. $M$ is called a $C R$ manifold of hypersurface type or $C R$ hypersurface for short if the real dimension of $T_{x}^{c} M:=T_{x} M \cap J T_{x} M$ is equal to $n$ for all $x \in M$, where $J$ is the standard complex structure of $\mathbb{C}^{N+1}$. Among CR hypersurfaces, the unit sphere $S$ is the simplest one. Moreover, it plays a role analogous to that of Euclidean space in Riemannian geometry as Chern and Moser emphasized in CM74.

One of the fundamental results in Riemannian geometry is the isometric embedding theorem into Euclidean spaces. It says that any smooth Riemannian manifold is locally embeddable into Euclidean spaces of sufficiently high dimension. But the corresponding theorem for CR geometry does not hold. In CR geometry, non-embeddable CR hypersurface into spheres is generic, as is seen in Far88 and Forst86. Moreover, there is a $C^{\omega} \mathrm{CR}$ hypersurface which is not embeddable into spheres of any (finite) dimension ( $[\mathrm{Z}]$ ). Recently, in $\mathrm{KO}$, the authors found necessary and sufficient conditions for a smooth CR hypersurface to be embeddable into a sphere in terms of Chern-Moser curvature tensors and their derivatives.

In this paper, we study CR hypersurfaces in $S$. One example is a transversal intersection of $S$ and a complex manifold. Conversely, one may ask whether any CR hypersurface in $S$ is a transversal intersection of $S$ and a complex manifold. In this paper we give a partial answer to this question.

Let $M$ be a germ of a CR hypersurface through $p$. A smooth tangent vector field on $M$ is called an infinitesimal CR automorphism if its flow generates a 1-parameter family of CR automorphisms. $M$ is said to be rigid if there exists an infinitesimal CR automorphism $X$ such that $X(p) \perp T_{p}^{c}(M)$. Our main result is the following.

Received by the editors October 12, 2009.

2010 Mathematics Subject Classification. Primary 32V30, 32V40; Secondary 53B25, 35N10.

This research was supported by the Basic Science Research Program through the National Research Foundation of Korea (NRF), funded by the Ministry of Education, Science and Technology (grant number 2009-0067947).

(C)2010 American Mathematical Society Reverts to public domain 28 years from publication 
Theorem 1. Let $M=M^{2 n+1}$ be a germ of a rigid CR hypersurface of dimension $2 n+1$ in a unit sphere $S=S^{2 N+1}$ of dimension $2 N+1$. Suppose $N-n<n / 2$. Then there exists a germ of a complex manifold $V$ of (complex) dimension $n+1$ intersecting $S$ transversally such that $M=S \cap V$. As a consequence, $M$ is real analytic. In particular, if $M^{2 n+1}$ is a rigid CR hypersurface in $S^{2 n+3}$ and if $n \geq 3$, then there exists a germ of a complex manifold $V$ of dimension $n$ intersecting $S$ transversally such that $M=S \cap V$. As a consequence, $M$ is real analytic.

If $M$ is not rigid, then there is a counterexample due to Huang. For a smooth function $f(w)=e^{-1 / w}$, the CR hypersurface $M$ defined by

$$
M=\left\{(z, w) \in \mathbb{C}^{2}: \operatorname{Im}(w)=|z|^{2}+|f(w)|^{2}\right\}
$$

is CR embeddable into $S^{5}$ but not real analytic.

The idea of the proof is to show that any nowhere-vanishing infinitesimal CR automorphism of $M$ in $S$ extends locally to an infinitesimal CR automorphism of $S$ and therefore is real analytically extendable. To show this, we use the rigidity of CR embeddings into spheres proved in Web79] and EHZ02. That is, any two germs of CR hypersurfaces in $S$ of sufficiently small codimension with equivalent CR structure can be mapped to each other by a CR automorphism of $S$. Since a nowhere-vanishing CR automorphism of $M$ extends real analytically to a neighborhood, $M$ is foliated by real analytic curves. Then by complexifying these curves, we extend the CR structure of $M$ to a complex structure of $M \times(\epsilon, \epsilon)$ to obtain the desired complex manifold $V$.

In $\S 1$, we review basic notions and the rigidity of CR embeddings into spheres. Then we state a further result (Theorem 4). In $\S 2$, we prove Theorem 4 . As a corollary, we prove Theorem 1.

Throughout this paper we use the summation convention with the following ranges for indices:

$$
\begin{array}{cl}
i, j, k \in\{0,1,2, \ldots, n, *\}, & I, J, K \in\{0,1,2, \ldots, N, *\}, \\
\alpha, \beta, \gamma \in\{1,2, \ldots, n\}, & a, b, c \in\{n+1, \ldots, N\} .
\end{array}
$$

We raise and lower indices of tensors using the matrix $\left(g_{I \bar{J}}\right)$, which will be defined in $\S 1$.

\section{Preliminaries AND A FURTher RESUlt}

In this section we review $\mathrm{CR}$ geometry and rigidity of $\mathrm{CR}$ embedding maps into spheres. Then we state a further result. We refer to [EHZ02] and [KO for details.

Let $S^{2 N+1}$ be a sphere in $\mathbb{C}^{N+1}$ defined by

$$
\sum_{m=1}^{N+1} z^{m} z^{\bar{m}}-1=0
$$

In homogeneous coordinates $z^{m}=\zeta^{m} / \zeta^{0}$, we may regard $S^{2 N+1}$ as a submanifold of $\mathbb{P}^{N+1}$ defined by $(\zeta, \zeta)=0$, where

$$
(\zeta, \zeta):=\sum_{m=1}^{N+1} \zeta^{m} \zeta^{\bar{m}}-\zeta^{0} \zeta^{\overline{0}}
$$

( , ) is a hermitian form of the signature $(N+1,1)$. 
A projective frame $\left\{Z_{I}\right\}=\left\{Z_{0}, \cdots, Z_{*}\right\}$ is a basis of $\mathbb{C}^{N+2}$ such that

$$
\operatorname{det}\left(Z_{0}, \cdots, Z_{*}\right)=+1 \text {. }
$$

At each point $p \in S^{2 N+1}$, we define $Z_{0}:=p$ to be the position vector and choose $Z_{*}$ satisfying

$$
\left(Z_{*}, Z_{*}\right)=0, \quad\left(Z_{0}, Z_{*}\right)=-i,
$$

so that $Z_{*}$ is a point in $S^{2 N+1}$ and a real tangent vector to $S^{2 N+1}$ at $p$. In addition to $Z_{0}$ and $Z_{*}$, we choose $N$ other linearly independent complex tangent vectors $Z_{A}$, $A=1, \ldots, N$, satisfying

$$
\left(Z_{0}, Z_{A}\right)=0, \quad\left(Z_{*}, Z_{A}\right)=0 .
$$

Such a frame shall be called an $S^{2 N+1}$-frame.

$S^{2 N+1}$-frames are unique up to a special unitary group $\mathbf{S U}(N+1,1)$ with respect to the form $(\zeta, \zeta)$. Define a Hermitian matrix $\left(g_{I \bar{J}}\right)$, where

$$
g_{I \bar{J}}:=\left(Z_{I}, Z_{J}\right)
$$

Then a transformation matrix $U$ from one frame to another satisfies $U g U^{*}=g$, where $g=\left(g_{I \bar{J}}\right)$. We may assume that for all $\alpha$ and $b$, it holds that

$$
g_{\alpha \bar{b}}=0
$$

and

$$
\left(g_{a \bar{b}}\right)=i d
$$

The Maurer-Cartan form $\pi:=\left(\pi_{I}^{J}\right)$ is defined by

$$
d Z_{I}=\pi_{I}^{J} Z_{J} \text {. }
$$

In view of (1.1) and (1.2), $\pi$ satisfies

$$
\pi_{I}^{K} g_{K \bar{J}}+g_{I \bar{K}} \pi_{\bar{J}}^{\bar{K}}=0, \quad \operatorname{tr} \pi=\pi_{I}^{I}=0 .
$$

For a smooth CR hypersurface $M^{2 n+1}$ in $S^{2 N+1}$, we can adapt an $S^{2 N+1}$-frame to $M^{2 n+1}$ to satisfy

$$
\left.d Z_{0}\right|_{M^{2 n+1}}=\pi_{0}^{0} Z_{0}+\pi_{0}{ }^{1} Z_{1}+\cdots+\pi_{0}^{n} Z_{n}+\pi_{0}{ }^{*} Z_{*} .
$$

From now on we only consider local embeddings. Choose 1 -forms $\left\{\omega^{\alpha}, \omega^{*}\right\}$ of $M^{2 n+1}$ such that $\left\{\omega^{\alpha}\right\}$ is a basis of type $(1,0)$-forms and $\omega^{*}$ is a real 1-form annihilating the complex tangent space $T^{c} M^{2 n+1}$ satisfying

$$
d \omega^{*}=i g_{\alpha \bar{\beta}} \omega^{\alpha} \wedge \omega^{\bar{\beta}}+\omega^{*} \wedge \varphi,
$$

where $\varphi$ is a real 1-form and $\left(g_{\alpha \bar{\beta}}\right)$ is a submatrix of $\left(g_{I \bar{J}}\right) .\left(g_{\alpha \bar{\beta}}\right)$ represents the Levi form of $M^{2 n+1}$. We can choose an adapted $S^{2 N+1}$-frame so that on $M^{2 n+1}$, it holds that

$$
\pi_{0}^{*}=\omega^{*}, \quad \pi_{0}^{\alpha}=\omega^{\alpha}, \quad \pi_{0}^{a}=0 .
$$

The exterior derivative of (1.3) gives the structure equation

$$
d \pi_{I}^{J}=\pi_{I}^{K} \wedge \pi_{K}^{J} .
$$

In particular, we have

$$
\begin{aligned}
0=d \pi_{0}{ }^{a} & =\pi_{0}{ }^{I} \wedge \pi_{I}{ }^{a} \\
& =\omega^{\beta} \wedge \pi_{\beta}{ }^{a}+\omega^{*} \wedge \pi_{*}{ }^{a} .
\end{aligned}
$$

Then by Cartan's lemma, we have

$$
\pi_{\alpha}^{a}=h_{\alpha \beta}^{a} \omega^{\beta}+k_{\alpha}^{a} \omega^{*}
$$


and

$$
\pi_{*}^{a}=k_{\alpha}^{a} \omega^{\alpha}+l^{a} \omega^{*}
$$

for some $h_{\alpha \beta}^{a}, k_{\alpha}^{a}, l^{a}$ with $h_{\alpha \beta}^{a}=h_{\beta \alpha}^{a}$. The coefficients $h_{\alpha \beta}^{a}$ form the second fundamental form

$$
I I:=h_{\alpha \beta}^{a} Z_{a} \omega^{\alpha} \otimes \omega^{\beta} .
$$

We define the covariant derivatives of $h_{\alpha \beta}^{a}, k_{\alpha}^{a}, l^{a}$ relative to $\pi$ by

$$
\begin{aligned}
D h_{\alpha \beta}^{a} & :=d h_{\alpha \beta}^{a}-\pi_{\alpha}^{\gamma} h_{\gamma \beta}^{a}-\pi_{\beta}^{\gamma} h_{\alpha \gamma}^{a}+\pi_{0}^{0} h_{\alpha \beta}^{a}+\pi_{b}{ }^{a} h_{\alpha \beta}^{b}, \\
D k_{\alpha}^{a} & :=d k_{\alpha}^{a}-\pi_{\alpha}^{\gamma} k_{\gamma}{ }^{a}+\left(\pi_{0}^{0}-\pi_{*}{ }^{*}\right) k_{\alpha}^{a}+\pi_{b}{ }^{a} k_{\alpha}^{b}-\pi_{*}{ }^{\beta} h_{\alpha \beta}^{a}, \\
D l^{a} & :=d l^{a}+\left(\pi_{0}{ }^{0}-2 \pi_{*}^{*}\right) l^{a}+\pi_{b}{ }^{a} l^{b}-2 \pi_{*}{ }^{\alpha} k_{\alpha}{ }^{a} .
\end{aligned}
$$

By differentiating (1.4) and (1.5), we obtain

$$
\begin{aligned}
& 0=d\left(\pi_{\alpha}^{a}-\left(h_{\alpha \beta}^{a} \omega^{\beta}+k_{\alpha}{ }^{a} \omega^{*}\right)\right)=-\left(D h_{\alpha \beta}^{a} \wedge \omega^{\beta}+D k_{\alpha}^{a} \wedge \omega^{*}+i k_{\alpha}{ }^{a} g_{\gamma \bar{\delta}} \omega^{\gamma} \wedge \omega^{\bar{\delta}}\right), \\
& 0=d\left(\pi_{*}{ }^{a}-\left(k_{\beta}{ }^{a} \omega^{\beta}+l^{a} \omega^{*}\right)\right)=-\left(D k_{\alpha}^{a} \wedge \omega^{\alpha}+D l^{a} \wedge \omega^{*}+i l^{a} g_{\gamma \bar{\delta}} \omega^{\gamma} \wedge \omega^{\bar{\delta}}\right) .
\end{aligned}
$$

Again by Cartan's lemma we obtain

$$
\begin{aligned}
D h_{\alpha \beta}^{a} & =h_{\alpha \beta ; \gamma}^{a} \omega^{\gamma}+h_{\alpha \beta ; \bar{\gamma}}^{a} \omega^{\bar{\gamma}}+h_{\alpha \beta ; *}^{a} \omega^{*}, \\
D k_{\alpha}^{a} & =k_{\alpha ; \gamma}^{a} \omega^{\gamma}+k_{\alpha ; \bar{\gamma}}^{a} \omega^{\bar{\gamma}}+k_{\alpha ; *}^{a} \omega^{*}, \\
D l^{a} & =l_{; \gamma}^{a} \omega^{\gamma}+l_{; \bar{\gamma}}^{a} \omega^{\bar{\gamma}}+l_{; *}^{a} \omega^{*},
\end{aligned}
$$

where

$$
\begin{aligned}
h_{\alpha \beta ; \gamma}^{a} & =h_{\alpha \gamma ; \beta}^{a}, h_{\alpha \rho ; \bar{\sigma}}^{a}=i\left(g_{\rho \bar{\sigma}} k_{\alpha}^{a}+g_{\alpha \bar{\sigma}} k_{\rho}^{a}\right), h_{\alpha \rho ; *}^{a}=k_{\alpha ; \rho}^{a}, \\
k_{\alpha ; \beta}^{a} & =k_{\beta ; \alpha}^{a}, k_{\rho ; \bar{\sigma}}^{a}=i g_{\rho \bar{\sigma}} l^{a}, k_{\alpha ; *}^{a}=l_{; \alpha}^{a}
\end{aligned}
$$

and

$$
l_{; \bar{\alpha}}^{a}=0 .
$$

Similarly, we define higher order covariant derivatives $D h_{\gamma_{1} \gamma_{2} ; \gamma_{3} \cdots \gamma_{j}}^{a}$ by

$$
\begin{aligned}
D h_{\gamma_{1} \gamma_{2} ; \gamma_{3} \cdots \gamma_{j}}^{a} & =d h_{\gamma_{1} \gamma_{2} ; \gamma_{3} \cdots \gamma_{j}}^{a}-\sum_{l=1}^{j} \pi_{\gamma_{l}}^{\mu} h_{\gamma_{1} \gamma_{2} ; \gamma_{3} \cdots \gamma_{l-1} \mu \gamma_{l+1} \cdots \gamma_{j}}^{a} \\
& +\pi_{0}{ }^{0} h_{\gamma_{1} \gamma_{2} ; \gamma_{3} \cdots \gamma_{j}}+\pi_{b}{ }^{a} h_{\gamma_{1} \gamma_{2} ; \gamma_{3} \cdots \gamma_{j}} .
\end{aligned}
$$

By Cartan's lemma we have

$$
D h_{\gamma_{1} \gamma_{2} ; \gamma_{3} \cdots \gamma_{j}}^{a}=h_{\gamma_{1} \gamma_{2} ; \gamma_{3} \cdots \gamma_{j} \mu}^{a} \omega^{\mu}+h_{\gamma_{1} \gamma_{2} ; \gamma_{3} \cdots \gamma_{j} \bar{\mu}}^{a} \omega^{\bar{\mu}}+h_{\gamma_{1} \gamma_{2} ; \gamma_{3} \cdots \gamma_{j} *}^{a} \omega^{*},
$$

where

$$
h_{\gamma_{1} \gamma_{2} ; \gamma_{3} \cdots \gamma_{j} \bar{\mu}}^{a}=h_{\gamma_{1} \gamma_{2} ; \gamma_{3} \cdots \bar{\mu} \gamma_{j}}^{a}+i g_{\gamma_{j} \bar{\mu}} h_{\gamma_{1} \gamma_{2} ; \gamma_{3} \cdots \gamma_{j-1} *}^{a}
$$

and

$$
h_{\gamma_{1} \gamma_{2} ; \gamma_{3} \cdots \gamma_{j} *}^{a}=h_{\gamma_{1} \gamma_{2} ; \gamma_{3} \cdots \gamma_{j-1} * \gamma_{j}}^{a} .
$$

By the same argument we can define $D k_{\gamma_{1} ; \gamma_{2} \cdots \gamma_{j}}^{a}$ and $D l_{; \gamma_{1} \cdots \gamma_{j}}^{a}$ with the condition

$$
k_{\gamma_{1} ; \gamma_{2} \cdots \gamma_{j} \bar{\mu}}^{a}=k_{\gamma_{1} ; \gamma_{2} \cdots \bar{\mu} \gamma_{j}}^{a}+i g_{\gamma_{j} \bar{\mu}} k_{\gamma_{1} ; \gamma_{2} \cdots \gamma_{j-1} *}^{a}, \quad k_{\gamma_{1} ; \gamma_{2} \cdots \gamma_{j} *}^{a}=k_{\gamma_{1} ; \gamma_{2} \cdots \gamma_{j-1} * \gamma_{j}}^{a}
$$

and

$$
l_{; \gamma_{1} \cdots \gamma_{j} \bar{\mu}}^{a}=l_{; \gamma_{1} \cdots \bar{\mu} \gamma_{j}}^{a}+i g_{\gamma_{j} \bar{\mu}} l_{; \gamma_{1} \cdots \gamma_{j-1} *}^{a} \quad l_{\gamma_{1} ; \cdots \gamma_{j} *}^{a}=l_{\gamma_{1} ; \cdots \gamma_{j-1} * \gamma_{j}}^{a} .
$$


Definition 1. For integers $k_{0}$ and $s_{0}$ with $k_{0} \geq 2, M^{2 n+1}$ in $S^{2 N+1}$ is said to be $\left(k_{0}, s_{0}\right)$-nondegenerate at $p$ if the dimension of the span of

$$
\left\{h_{\gamma_{1} \gamma_{2} ; \gamma_{3} \cdots \gamma_{j}}^{a} Z_{a}, 2 \leq j \leq k\right\}
$$

at $p$ is $N-n-s_{0}$ for $k \geq k_{0}$ and strictly smaller for $k<k_{0}$. The smallest $s_{0}$ as $p$ varies is called the degeneracy of $M^{2 n+1}$. If $M^{2 n+1}$ is of 0 -degeneracy, then $M^{2 n+1}$ is said to be finitely nondegenerate. Degeneracy of a germ $M^{2 n+1}$ is defined to be the largest $s$ such that $M^{2 n+1}$ has a connected representation of $s$-degeneracy.

In [EHZ02, the following two theorems are proved.

Theorem 2. Let $M^{2 n+1}$ be a connected smooth CR hypersurface and let $f$ : $M^{2 n+1} \rightarrow S^{2 N+1}$ be a smooth $C R$ embedding such that the degeneracy of the image $f\left(M^{2 n+1}\right)$ is s. If $N-n-s<n$, then there exists a complex plane $P$ of codimension $s$ in $\mathbb{C}^{N+2}$ such that $f\left(M^{2 n+1}\right)$ is contained in $S^{2 N+1} \cap P$.

Theorem 3. Let $f: M^{2 n+1} \rightarrow S^{2 N+1}$ be as in Theorem 2. If $N-n-s<n / 2$, then $f$ is rigid. That is, any other such $C R$ embedding $\tilde{f}: M^{2 n+1} \rightarrow S^{2 N+1}$ is related to $f$ by $\tilde{f}=\phi \circ f$, where $\phi$ is a CR automorphism of $S^{2 N+1}$.

Now we state our main theorem.

Theorem 4. Let $M=M^{2 n+1}$ be a germ of a rigid CR hypersurface in $S=S^{2 N+1}$ with $s$-degeneracy. Suppose that $N-n-s<n / 2$. Then there exists a germ of a complex manifold $V$ of dimension $n+1$ intersecting $S$ transversally such that $M=S \cap V$. As a consequence, $M^{2 n+1}$ is real analytic.

\section{Proof of the main theorem}

Let $M=M^{2 n+1}$ be a germ of a smooth CR hypersurface through $p$ in $S=S^{2 N+1}$ with $s$-degeneracy such that $N-n-s<n / 2$. By Theorem 2 , there exists a complex plane $P$ of codimension $s$ such that $M \subset S \cap P$. By replacing $S$ by $S \cap P$, we may assume that $M$ is finitely nondegenerate in $S^{2(N-s)+1}$.

From now on we assume that $M$ is finitely nondegenerate in $S$ with $N-n<n / 2$. Assume further that $M$ is rigid. Choose an infinitesimal CR automorphism $X$ of $M$ such that $X(p) \perp T_{p}^{c}(M)$. We assume that $M$ and $X$ are well defined in a suitable open neighborhood of $p$ and $X$ is nowhere vanishing. Let $\left\{f_{t}\right\}$ be the 1-parameter family of CR automorphisms of $M$ such that

$$
\begin{aligned}
f_{0} & =i d, \\
\frac{\partial}{\partial t}\left(f_{t}\right) & =X \circ f_{t} .
\end{aligned}
$$

By Theorem 3, we can choose a 1-parameter family of CR automorphisms $\left\{\phi_{t}\right\}$ of $S$ such that $f_{t}=\phi_{t} \circ f_{0}$.

Lemma 1. $\phi_{0}=i d$ and $\phi_{t}$ depends smoothly on $t$.

Proof. Choose an open set $\mathcal{U}$ in $M$ and an integer $k_{0} \geq 2$ such that on $\mathcal{U}$ we have

$$
\begin{aligned}
\operatorname{span}\left\{h_{\gamma_{1} \gamma_{2}}^{a} Z_{a}\right\} & <\operatorname{span}\left\{h_{\gamma_{1} \gamma_{2} ; \gamma_{3}}^{a} Z_{a}\right\} \\
& <\cdots \\
& <\operatorname{span}\left\{h_{\gamma_{1} \gamma_{2} ; \gamma_{3} \cdots \gamma_{j}}^{a} Z_{a}, 2 \leq j \leq k_{0}\right\}=\operatorname{span}\left\{Z_{a}\right\} .
\end{aligned}
$$


Choose an $S$-frame adapted to $M$ and integers $r_{2}<\cdots<r_{k_{0}}=N$ such that on $\mathcal{U}$, it holds that for $k \leq k_{0}$,

$$
\operatorname{span}\left\{h_{\gamma_{1} \gamma_{2} ; \gamma_{3} \cdots \gamma_{j}}^{a} Z_{a}, 2 \leq j \leq k\right\}=\operatorname{span}\left\{Z_{a}, a=n+1, \ldots, r_{k}\right\} .
$$

Note that on $\mathcal{U}$ we have

$$
\operatorname{span}\left\{\phi_{t *}\left(Z_{a}\right), a=n+1, \ldots, r_{k}\right\}=\operatorname{span}\left\{Z_{a}, a=n+1, \ldots, r_{k}\right\} .
$$

Since $f_{t}=\phi_{t} \circ f$, where $\phi_{t}$ is an automorphism of $S$, there exists a 1-parameter family of matrix $U_{t}=\left(U_{t I}^{J}\right)$ satisfying $U_{t} g U_{t}^{*}=g$ such that

$$
\phi_{t}^{*}\left(\pi_{I}^{J}\right)=\pi_{I}^{K} U_{t K}^{J}
$$

where $\left(\pi_{I}^{J}\right)$ is the Maurer-Cartan form of $S$. To prove the lemma, it is enough to show that $U_{0}=i d$ and $U_{t}$ depends smoothly on $t$.

Fix $t$ and let $I I=h_{\alpha \beta}^{a} Z_{a} \omega^{\alpha} \otimes \omega^{\beta}$ be the second fundamental form of $M$. Then we have

$$
h_{t \alpha \beta}^{a}(x)=h_{\alpha \beta}^{a}\left(f_{t}(x)\right)=h_{\alpha \beta}^{b} U_{t b}^{a}(x),
$$

where $h_{t \alpha \beta}^{a} Z_{a} \omega^{\alpha} \otimes \omega^{\beta}$ is the second fundamental form of $\phi_{t}(M)$. We may assume that $f_{t}(\mathcal{U}) \cap \mathcal{U} \neq \emptyset$. Since $t$ is fixed, we denote $U_{t}$ by $\left(U_{a}{ }^{b}\right)$ for simplicity. Define the covariant derivative $D U_{a}{ }^{b}$ by

$$
D U_{a}^{b}=d U_{a}^{b}-U_{a}^{c} \pi_{c}^{b}-U_{c}^{b} \pi_{a}^{c} .
$$

By Cartan's lemma we can show that

$$
D U_{a}^{b}=U_{a: \alpha}^{b} \omega^{\alpha}+U_{a: \bar{\alpha}^{b}} \omega^{\bar{\alpha}}+U_{a: *}^{b} \omega^{*} .
$$

We claim that for any $j$,

$$
\begin{aligned}
h_{\alpha \beta ; \gamma_{1} \cdots \gamma_{j}}^{a} U_{a ; \rho}^{b} & =h_{\alpha \beta ; \gamma_{1} \cdots \gamma_{j}}^{a} U_{a ; \bar{\rho}}^{b}=0, \\
k_{\alpha ; \gamma_{1} \cdots \gamma_{j}}^{a} U_{a ; \rho}^{b} & =k_{\alpha ; \gamma_{1} \cdots \gamma_{j}}^{a} U_{a ; \bar{\rho}}^{b}=0, \\
l_{; \gamma_{1} \cdots \gamma_{j}}^{a} U_{a ; \rho}^{b} & =l_{; \gamma_{1} \cdots \gamma_{j}}^{a} U_{a ; \bar{\rho}}^{b}=0 .
\end{aligned}
$$

To prove the claim, we will use induction on $j$. Let $j=0$. Differentiate the equation

$$
h_{t \alpha \beta}^{a}=h_{\alpha \beta}^{b} U_{b}^{a}
$$

to obtain

$$
h_{t \alpha \beta ; \bar{\rho}}^{a}=h_{\alpha \beta ; \bar{\rho}}^{b} U_{b}^{a}+h_{\alpha \beta}^{b} U_{b ; \bar{\rho}}^{a},
$$

where $h_{\alpha \beta}^{a}\left(f_{t}\right)=h_{t \alpha \beta}^{a}$. Since

$$
h_{\alpha \rho ; \bar{\sigma}}^{a}=i\left(g_{\rho \bar{\sigma}} k_{\alpha}^{a}+g_{\alpha \bar{\sigma}} k_{\rho}^{a}\right)
$$

and since

where $k_{t \alpha}^{a}=k_{\alpha}^{a}\left(f_{t}\right)$, we obtain

$$
k_{t \alpha}^{a}=k_{\alpha}^{b} U_{b}^{a},
$$

$$
h_{\alpha \beta}^{b} U_{b ; \bar{\rho}}^{a}=0 .
$$

By differentiating $U g U^{*}=g$, we obtain

$$
U_{a ; \bar{\rho}}^{b} \bar{U}_{b}^{c}+U_{a}^{b} \bar{U}_{b ; \bar{\rho}}^{c}=0 .
$$

Multiply $h_{\alpha \beta}^{a}$ to obtain

$$
h_{\alpha \beta}^{a} U_{a}^{b} \bar{U}_{b ; \bar{\rho}}^{c}=0 .
$$


Multiply $h_{\bar{\gamma} c \bar{\delta}}$ to obtain

$$
h_{\alpha \beta}^{a} U_{a}^{b} \bar{U}_{b ; \bar{\rho}}^{c} h_{\bar{\gamma} c \bar{\delta}}=0 .
$$

Then by (2.1), we can show that

$$
h_{\alpha \beta}^{a} U_{a ; \rho}^{b}=0 .
$$

Also, by a similar argument we can show that

$$
k_{\alpha}^{a} U_{a ; \rho}^{b}=k_{\alpha}^{a} U_{a ; \bar{\rho}}^{b}=0
$$

and

$$
l^{a} U_{a ; \rho}^{b}=l^{a} U_{a ; \bar{\rho}}^{b}=0
$$

which completes the proof for $j=0$. Note that this yields that

$$
h_{t \alpha \beta ; \gamma}^{a}=h_{\alpha \beta ; \gamma}^{b} U_{b}^{a}, k_{t \alpha ; \gamma}^{a}=k_{\alpha ; \gamma}^{b} U_{b}^{a}, l_{t ; \gamma}^{a}=h_{; \gamma}^{b} U_{b}^{a} .
$$

Hence by the same argument, we can complete the induction argument for the claim.

Since

we have

$$
h_{\alpha \beta ; \gamma_{1} \cdots \gamma_{j}}^{a} U_{a ; \rho}^{b}=0,
$$

$$
h_{t \alpha \beta ; \gamma_{1} \cdots \gamma_{j}}^{a}=h_{\alpha \beta ; \gamma_{1} \cdots \gamma_{j}}^{b} U_{b}^{a} .
$$

Since $h_{t \alpha \beta}^{a}$ and their derivatives depend smoothly on $t$, by (2.1) we can show that $U_{t a}^{b}$ depends smoothly on $t$. Since $f_{t}$ depends smoothly on $t$, the complex tangent space $T_{f_{t}(x)}^{1,0} M$ depends smoothly on $t$ for fixed $x \in \mathcal{U}$. Therefore we have that $U_{0}=i d$ and that $U_{t}$ depends smoothly on $t$.

Now consider a 1-parameter family of CR automorphisms $\left\{\phi_{t}\right\}$. Then by differentiation, we obtain an infinitesimal CR automorphism $\widetilde{X}$ of $S$. It can be seen from the Chern-Moser theory (CM74]) that any nonvanishing infinitesimal CR automorphism of $S$ is real analytic. Therefore, after shrinking a neighborhood if necessary, we may assume that $\widetilde{X}$ is written as

$$
\widetilde{X}=R e\left(\sum_{j=1}^{N+1} h^{j} \frac{\partial}{\partial z_{j}}\right),
$$

where $h^{j}, j=1, \ldots, N+1$, are holomorphic functions ([BER99]). Let

$$
Y:=\sum_{j=1}^{N+1} h^{j} \frac{\partial}{\partial z_{j}} .
$$

Since $X(p)$ is transverse to $T_{p}^{c}(M)$, we see that $\operatorname{Im}(Y)$ is transverse to TS near $p$.

Since $f_{0}=i d$ and $f_{t}$ maps $M$ to $M$, we can show that $\left.\tilde{X}\right|_{M}=X$. Therefore $M$ is foliated by real analytic curves $c(t)$ with $\dot{c}(t)=\widetilde{X}(c(t))$. For each curve $c$, choose the unique holomorphic curve $D_{c}(w)$ by complexifying $c(t)$, where $w=t+\sqrt{-1} s$. Then we can show that

$$
\frac{d}{d w} D_{c}(w)=Y\left(D_{c}(w)\right)
$$

Since $\operatorname{Im}(Y)$ is transverse to $S$, we have $D_{c} \cap S=c$.

Now set $V=\bigcup_{c} D_{c}$. Obviously we have $M=V \cap S$. Moreover, near $p, V$ is a smooth manifold of real dimension $2 n+2$ and intersects $S$ transversally. Then the next lemma will complete the proof. 
Lemma 2. $V$ is a complex manifold.

Proof. The proof of the lemma is a modification of straightening infinitesimal the $\mathrm{CR}$ automorphisms transversal to the $\mathrm{CR}$ structure given in [BR].

Assume that $p=0$. Choose a smooth submanifold $U \subset M$ of dimension $2 n$ containing 0 and a complex disc $\Delta_{\epsilon} \subset \mathbb{C}$ of radius $\epsilon$ such that $T_{0}(U)=T_{0}^{c}(M)$ and the map $\Phi$ defined by

$$
(x, w) \in U \times \Delta_{\epsilon} \rightarrow D_{c_{x}}(w) \in V
$$

is a smooth diffeomorphism onto an open set of $V$ containing 0 , where $c_{x}$ is the unique integral curve of $X$ such that $c_{x}(0)=x$. By definition, $\Phi$ restricted to $U \times(\epsilon, \epsilon)$ is a diffeomorphism onto an open set of $M$ containing 0 and $\Phi_{*}\left(\frac{\partial}{\partial t}\right)=X$, where $t=\operatorname{Re}(w)$.

Choose linearly independent smooth vector fields $L_{1}, \ldots, L_{n}$ on $U \times(-\epsilon, \epsilon)$ such that $\Phi_{*}\left(L_{1}\right), \ldots, \Phi_{*}\left(L_{n}\right)$ are CR vector fields of $M$. We may assume that

$$
L_{j}=\frac{\partial}{\partial z_{j}}+\sum_{j=1}^{n} a_{j}^{i}(x, t) \frac{\partial}{\partial \bar{z}_{i}}+b_{j}(x, t) \frac{\partial}{\partial t}, j=1, \ldots, n,
$$

where we use $(x, t)=\left(x_{1}, \ldots, x_{2 n}, t\right)$ as coordinates of $U \times(-\epsilon, \epsilon)$ and $z_{j}=x_{2 j-1}+$ $\sqrt{-1} x_{2 j}$. Since $X(0)$ is transverse to $T_{0}^{c} M$, we have $b_{j}(0)=0$ for all $j=1, \ldots, n$. Furthermore, we may assume that $a_{j}^{i}(0)=0$ for all $i, j=1, \ldots, n$ and that

$$
\left[L_{j}, L_{k}\right] \equiv 0, j, k=1, \ldots, n .
$$

Since $X$ is an infinitesimal CR automorphism, we have $\left[\Phi_{*}\left(L_{j}\right), X\right]=0$ modulo $\Phi_{*}\left(L_{j}\right), j=1, \ldots, n$, which yields

$$
\frac{\partial}{\partial t}\left(a_{j}^{i}\right) \equiv \frac{\partial}{\partial t}\left(b_{j}\right) \equiv 0, i, j=1, \ldots, n .
$$

That is, $a_{j}^{i}$ and $b_{j}$ are independent of $t$. Now extend $L_{j}, j=1, \ldots, n$, to $U \times \Delta_{\epsilon}$ by

$$
\widetilde{L}_{j}(x, w):=\frac{\partial}{\partial z_{j}}+\sum_{j=1}^{n} a_{j}^{i}(x) \frac{\partial}{\partial \bar{z}_{i}}+b_{j}(x) \frac{\partial}{\partial t}, j=1, \ldots, n,
$$

where $w=t+\sqrt{-1} s$. Then we can see that

$$
\left[\widetilde{L}_{j}, \widetilde{L}_{k}\right] \equiv\left[\widetilde{L}_{j}, \frac{\partial}{\partial w}\right] \equiv 0
$$

By the Newlander-Nirenberg Theorem, $U \times \Delta_{\epsilon}$ is a complex manifold of (complex) dimension $n+1$.

We will show that $\Phi$ is a holomorphic map into $\mathbb{C}^{N+1}$. To show this, it is enough to show that $\Phi_{*}\left(\widetilde{L}_{j}\right), j=1, \ldots, n$, and $\Phi_{*}\left(\frac{\partial}{\partial w}\right)$ are $(1,0)$ vector fields in $\mathbb{C}^{N+1}$.

Clearly $\Phi_{*}\left(\frac{\partial}{\partial w}\right)=Y$ and hence is a $(1,0)$ vector field in $\mathbb{C}^{N+1}$. Let $g_{s}$ be a 1-parameter family of diffeomorphism of $U \times \Delta_{\epsilon}$ satisfying

$$
\begin{aligned}
g_{0} & =i d, \\
\frac{\partial}{\partial s}\left(g_{s}\right) & =\frac{\partial}{\partial s},
\end{aligned}
$$


where $s=\operatorname{Im}(w)$. Then by the definition of $\widetilde{L}_{j}$, we can show that

$$
\widetilde{L}_{j}(x, w)=\left(g_{s}\right)_{*}\left(L_{j}(x, t)\right),
$$

where $w=t+\sqrt{-1} s$. Let $G_{s}:=\Phi \circ g_{s} \circ \Phi^{-1}$. Then $G_{s}$ maps $V$ to $V$ and

$$
\begin{aligned}
G_{0} & =i d, \\
\frac{\partial}{\partial s}\left(G_{s}\right) & =\operatorname{Im}\left(Y\left(G_{s}\right)\right) .
\end{aligned}
$$

Since $Y$ is a holomorphic vector field in $\mathbb{C}^{N+1}$, for each small $s, G_{s}$ is the restriction of a holomorphic map to $V$. Since $\Phi_{*}\left(L_{j}\right)$ is a $(1,0)$ vector field on $M$ and since

$$
\Phi_{*}\left(\widetilde{L}_{j}(x, w)\right)=\Phi_{*} \circ\left(g_{s}\right)_{*}\left(L_{j}(x, t)\right)=\left(G_{s}\right)_{*} \circ \Phi_{*}\left(L_{j}(x, t)\right),
$$

we can see that $\Phi_{*}\left(\widetilde{L}_{j}\right), j=1, \ldots, n$, are $(1,0)$ vector fields in $\mathbb{C}^{N+1}$.

\section{ACKNOWLEDGMENTS}

The author thanks Professor Xiaojun Huang for valuable discussions during his visit to Seoul National University.

\section{REFERENCES}

[BER99] Baouendi, M. S., Ebenfelt, P. and Rothschild, L. P., Real Submanifolds in Complex Space and Their Mappings, Princeton Math. Series 47, Princeton University Press, New Jersey, 1999. MR1668103 (2000b:32066)

[BR] Baouendi, M. S., Rothschild, L. P. and Treves, F., CR structures with group action and extendability of CR functions, Invent. Math. 82 (1985), no. 2, 359-396. MR809720 (87i:32028)

[CM74] Chern, S. S., and Moser, J. K., Real hypersurfaces in complex manifolds, Acta Math. 133 (1974), 219-271. MR0425155 (54:13112)

[EHZ02] Ebenfelt, P., Huang, X. and Zaitsev, D., Rigidity of CR-immersions into spheres, Comm. Anal. Geom. 12 (2004), no. 3, 631-670. MR2128606 (2006c:53037)

[Far88] Faran, J., The nonembeddability of real hypersurfaces in spheres, Proc. Amer. Math. Soc. 103 (1988), 902-904. MR947678 (89i:32037)

[Forst86] Forstnerič, F., Embedding strictly pseudoconvex domains into balls, Trans. Amer. Math. Soc. 295 (1986), 347-368. MR831203 (87k:32052)

[KO] Kim, S.-Y. and Oh, J.-O., Local embeddability of CR manifolds into spheres, Math. Ann. 344 (2009), no. 1, 185-211. MR2481058 (2010h:32048)

[Web79] Webster, S. M., The rigidity of $C$ - $R$ hypersurfaces in a sphere, Indiana Univ. Math. J. 28 (1979), 405-416. MR.529673 (80d:32022)

[Z] Zaitsev, D., Obstructions to embeddability into hyperquadrics and explicit examples, Math. Ann. 342 (2008), no. 3, 695-726. MR2430996 (2009g:32073)

Department of Mathematics Educhtion, Kangwon National University, 123 HyojaDOnG, Chuncheon, KAngwon-Do, 200-701, Korea

E-mail address: sykim87@kangwon.ac.kr 